Bulgarian Academy of Sciences. Space Research and Technology Institute.

Aerospace Research in Bulgaria. 33, 2021, Sofia

DOI: https://doi.org/10.3897/arb.v33.e13

\title{
AERODYNAMIC PERFORMANCE OF CHEVRON GEOMETRY TRAILING EDGE FOR A TRANSONIC AXIAL COMPRESSOR IMPELLER
}

\author{
Ioana Octavia Bucur ${ }^{1}$, Ion Malael ${ }^{1}$, Valeriu Dragan ${ }^{1}$ \\ ${ }^{I}$ National Research and Development Institute for Gas Turbines COMOTI, 220D Iuliu \\ Maniu Bd., sector 6, cod 061126, OP 76, CP174, Bucharest, Romania. \\ e-mail: ioana.bucur@comoti.ro
}

Key words: Attenuate Noise Level, Transonic Axial Compressor, Chevron Geometry

\begin{abstract}
Several studies present how serrated rotor blades mix wakes in order to attenuate noise levels. The current paper analyses how this geometry, applied on the trailing edge, affects the global parameters of a transonic axial compressor impeller. Innovative solutions tackling the rotor-stator interaction mechanism in an axial compressor for noise reduction include serrated trailing edges. Inspired by chevron nozzles, serrations can be transferred to the open-rotor concept in order to reduce tonal noise. Throughout the study we will be focusing on aerodynamic loss estimation while being mindful of the mechanisms which lead to rotor-stator interaction noise, without assessing its per-se effectiveness for noise mitigation. Owing to its qualitative experimental data availability, NASA's Rotor 37 was chosen as a baseline. A set of fully viscous $3 D$ simulations, using the SST $k$-omega turbulence model and RANS, was carried out to this effect. Spatial discretization was made using a fully structured pre-mesh in order to optimize resolution and accelerate convergence. Fullfactorial samples were generated for the geometric variations in order to capture the aerodynamic implications of this concept. Overall, the analysed case provides promising perspectives, pending optimization studies and experimental tests thereof.
\end{abstract}

\section{Introduction}

Current concerns regarding the state of the art referring to transonic axial compressors mainly relates to optimization solutions and development ideas that can help in engine downsizing, without adversely impacting performances [1]. Understanding flow phenomena through the axial compressor is of vital importance in the context of modern growth, as most optimization methods rely on flow control through various methods (for example design modifications) [2].

Blade optimization represents a problem of interest in the aerospace engineering field as it may consistently improve overall performances of the propulsion system and its components. Hence, there are multiple innovative 
approaches considering blade improvement that can prevent loss and malfunction in axial compressors [3] and many recent studies concentrate on blade shape optimization using CFD methods.

A well-known and largely used transonic axial compressor test case for optimization is NASA's Rotor 37. First, it was tested as a single stage by Lonnie Reid and Royce D. Moore in 1978 [4], then again in 1980, this time as an isolated component [5] and so its geometry began to be identified by NASA and other developers in the field as Rotor 37 [6].

This test case is still a present topic and represents an important evaluation base in terms of overall performances and parameters for studies using CFD codes and other modern computational methods. Some researches based on the Rotor 37 case are $[6,7]$. Centralized data for Rotor 37 , including overall performances and parameters of interest, is presented in Tab. 1.

Table 1. Design Overall performance parameters for Rotor 37, [5]

\begin{tabular}{|c|r|}
\hline Parameters & Value \\
\hline Rotor total pressure ratio & 2.106 \\
\hline Rotor total temperature ratio & 1.270 \\
\hline Rotor adiabatic efficiency & 0.877 \\
\hline Rotor polytropic efficiency & 0.889 \\
\hline Rotor head rise coefficient & 0.333 \\
\hline Flow coefficient & 0.453 \\
\hline Airflow per unit frontal area & 100.950 \\
\hline Airflow per unit annulus area & 200.549 \\
\hline Airflow [kg/s] & 20.188 \\
\hline RPM & 17188.700 \\
\hline Tip speed [m/s] & 454.136 \\
\hline Hub-tip radius ratio & 0.70 \\
\hline Rotor aspect ratio & 1.19 \\
\hline Number of rotor blades & 36 \\
\hline
\end{tabular}

In paper [8] is presented an attempt to create an optimized design for the Rotor $37 \mathrm{~s}$ blade. The proposed shape for the blade aimed to slightly improve aerodynamic performances compared to the results provided by NASA's technical reports mentioned above.

In the context of modern development, studies have been carried out concerning optimization solutions for rotor blades. Those address both overall performances of the rotor and also mitigation of tonal sound or broadband sound that occurs in the compressor's rotor [9].

General Electric brings an innovative proposal that consists of using serrated fan blades in order to attenuate noise levels. This technique should not have a negative impact on the effectiveness of the engine or its performances, they 
claim [10]. For a better understanding of their invention, Fig. 1 is provided from the original patent.

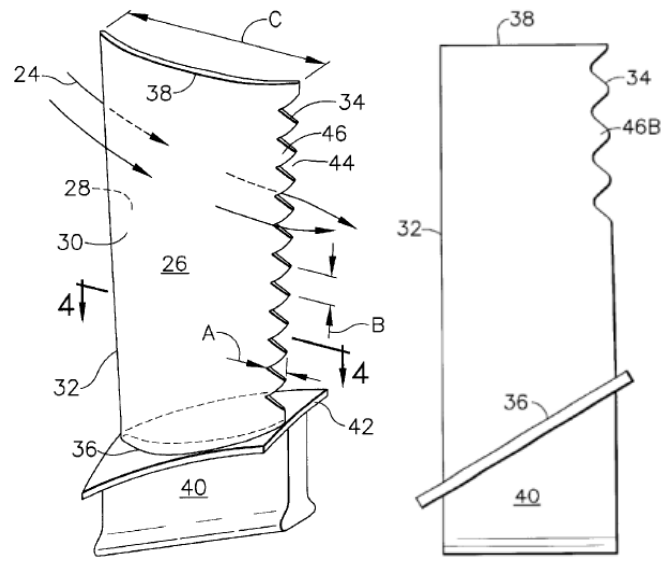

Fig. 1. Isometric and side view of an isolated fan blade embodiment [10]

The flow through an axial fan stage is analyzed by J. M. Fernández et al. in paper [11], using a LES based simulation to assess better understanding of rotorstator interaction in the fan stage.

Jet engines have benefited from chevron technology for noise reduction ever since its advent. Since the successful implementation of chevrons for turbofan engines, other jet related applications arose such as jet pumps for ventilation $[12,13]$.

Hence, several studies exploited chevron patterns in order to obtain solutions regarding noise attenuation for a sustainable development.

For example, trailing edge serrations were used in the improvement of an open-rotor. Their purpose was to mitigate tonal interaction noise. The results of steady-state RANS simulations concluded that this approach was successful in providing acoustical optimization [14].

Another recent study analysed the effects of leading edge serrations, taking into consideration overall aerodynamic performances and the impact on noise levels. For the tests conducted a wind tunnel was used. This research concluded that leading edge serrations can provide the attenuation of turbulence-interaction broadband noise when they are carefully optimized. Furthermore, there is introduced the concept of curved-serrations. The authors claim that their study can serve as a baseline in future attempts to develop new techniques in the area of noise control [15].

In the current paper, the main purpose is to study the influence of trailing edge blade serrations on a rotor impeller. To this extent NASA's Rotor 37 is used as a baseline, as its geometry is available for such test cases. 3D viscous 
simulations are carried out on the baseline geometry and three modified configurations, using the commercial CFD software Ansys. Details regarding the mesh and the used scheme are presented in the next section. Regarding the chevron characteristics, they are distributed along one third of the upper side of the blade and several configurations are studied: with two, three and four chevrons, distributed equally on the mentioned region. This approach allows assessing insightful information about how the number of serrations can affect the global performances of the rotor.

\section{CFD Setup}

Two batteries of CFD cases are carried out in order to assess chevron influence on aerodynamic behavior of the high performance transonic axial compressor. Four geometries were considered: the baseline plus three altered chevroned trailing edge blades. A minimum of two chevrons were cut from the original blade progressively, offset from the tip of the blade, being placed on one third of the upper side of the trailing edge. The other two geometries altered only the named section, but this time with three and four chevrons.

In order to make the cases comparable, the same mesh topology (Fig. 2 right) was used for each geometry, hence a cutoff trailing edge was opted for. Also, in order to eliminate as much of the secondary flow patterns in that region as possible, the geometry was considered without the tip gap - hence the rotor was treated as a shrouded compressor.

Care was taken so that the first element near the wall is consistent with the requirements for k-omega SST turbulence model used. Also, quality wise, the skewness as well as aspect and growth ratios were evaluated and made to fit within the best practice guidelines. The adopted value for $y^{+}$was 1 and the growth ratio was no more than 1.1. Regarding the skewness, a minimum of $20^{\circ}$ was set.

The second order upwind scheme was employed in all cases to insure the greatest accuracy of the model. Air was considered an ideal fluid and the inlet conditions were ISA total pressure for all simulations.

The first battery consisted of a greater than optimal outlet constant mass flow of $20.38 \mathrm{~kg} / \mathrm{s}$ (compared to the $20.188 \mathrm{~kg} / \mathrm{s}$ value given by NASA's technical report in Tab. 1). The decision was taken to study this regime because it will bring more information to the overall essay regarding a sensitive region of the compressor map. Functionally, the compressor operates with a nominal static backpressure; hence the second battery of tests was carried out with static outlet pressure of $1.1 \mathrm{~atm}$.

In Fig. 2, the meridional view of the blade with four chevrons is presented on the left. The baseline and the other two configurations (with two and three chevrons) are not illustrated, as the geometry with four chevrons was considered to be the most relevant in serving the purpose of highlighting the area of interest. 

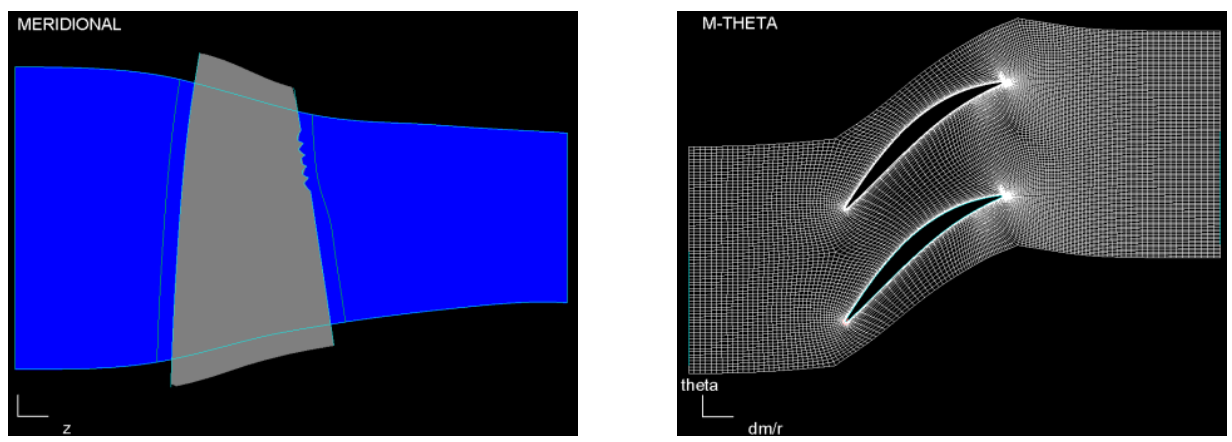

Fig. 2. Meridional view (left) and blade to blade topology of the mesh used (right)

\section{Results}

Regarding the $20.38 \mathrm{~kg} / \mathrm{s}$ batch, for the baseline the isentropic efficiency is $76.54 \%$ and the pressure ratio 1.644 compared to the values given in Table 1 by NASA of $87.7 \%$ for the isentropic efficiency, 2.106 for the pressure ratio respectively.

After synthesizing the constant mass flow cases, we have observed a clear decrease in isentropic efficiency of the modified stages along with a less dramatic decrease in total pressure ratio. In Fig. 3 one can see that the slight decrease in power consumption of the compressor is in spite of a dramatic decrease in isentropic efficiency and due to a decrease in delivered mechanical work.

An oddity is that the case with 4 chevrons appears to behave better than the rest of the modified geometries. This indicates that optimal placement and span of chevrons is not intuitive and must be tested further.

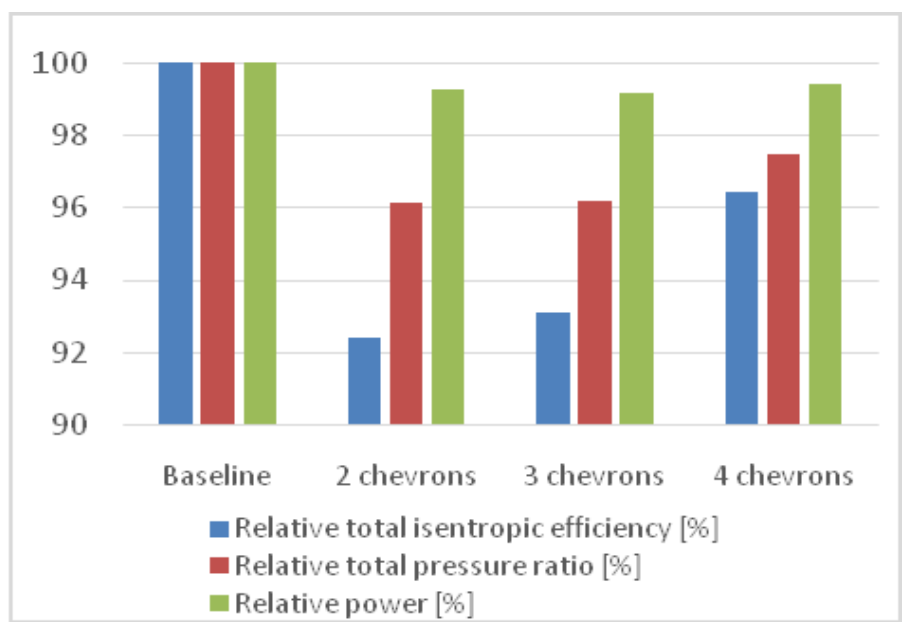

Fig. 3. Comparison between the functional parameters of the $20.38 \mathrm{~kg} / \mathrm{s}$ batch 


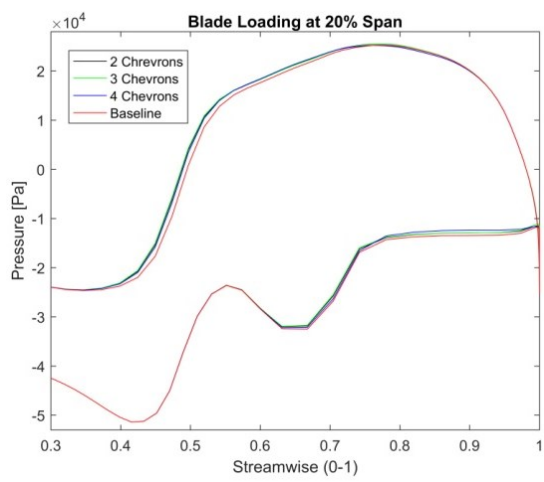

a)

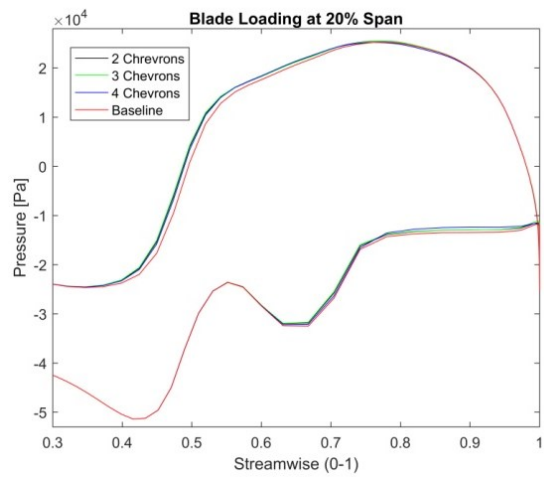

b)

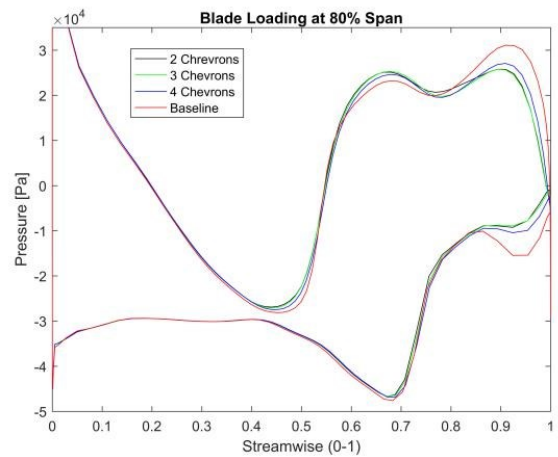

c)

Fig. 4. Blade loading across the span of the blade: a) 20\% Span, b) 50\% Span, c) $80 \%$ Span 
In the plots of Fig. 4, one can see the blade loading at $20 \%, 50 \%$ and $80 \%$, for all cases at every span, the loading is slightly lower than the baseline. It is interesting that even near the hub, where the chevrons are not present, the loading is lower.

On the other hand, there is no lowering of the overall efficiency as seen when analyzing the downstream entropy generation (Fig. 5 - left) or the evolution of isentropic efficiency across streamwise locations (Fig. 5 - right). Due to the vortical structures induced by the chevrons, part of the total pressure is converted to heat.
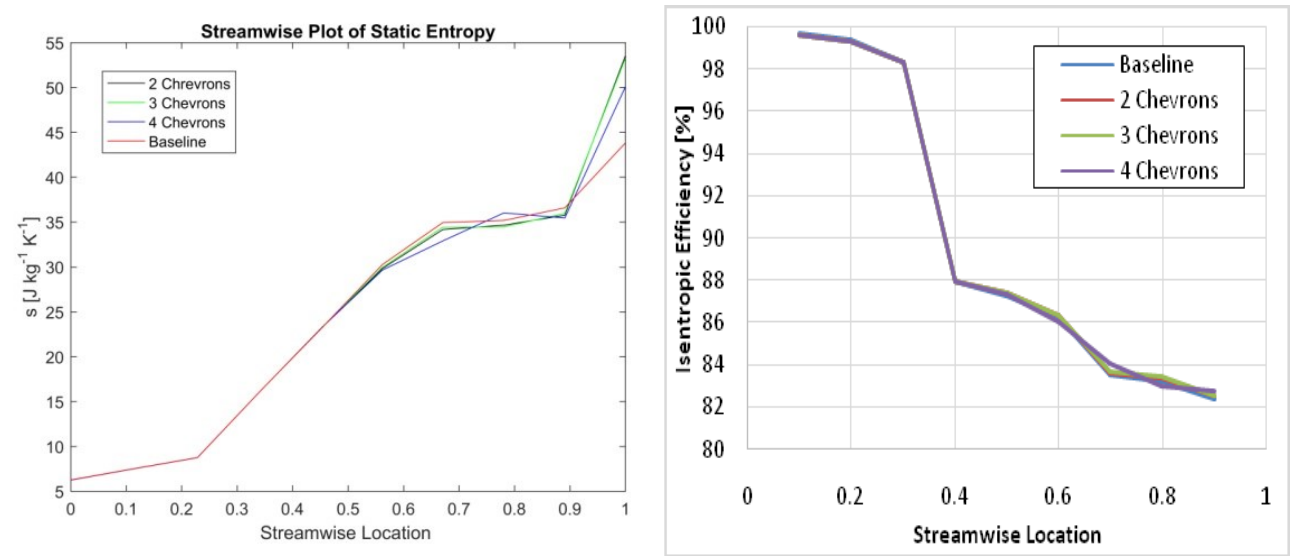

Fig. 5. Entropy across the blade passage (left) and isentropic efficiency evolution (right)

Note that the measurements on the outlet boundary, at streamwise location 1 , is not relevant because of boundary effects and should not be considered for analysis.

The second batch of tests refers to the static pressure outlet of $1.1 \mathrm{~atm}$. In this case, when comparing the values of the isentropic efficiency $(84.77 \%)$ and pressure ratio $(1.89 \mathrm{~kg} / \mathrm{s})$ for the baseline with the data provided in Tab. 1 by NASA $(87.7 \%, 2.106$ respectively), the accuracy is better than in the previous discussed case.

One can see in Fig. 6 that the relative power consumption is no longer linear with the number of chevrons. This is because all parameters vary independently; therefore each case has a different mass flow, total pressure ratio and total efficiency.

Although difficult to interpret under these circumstances, the main conclusion is that the ability to compress the fluid is diminished proportionately to the span of the chevroned portion. This has little or no bearing on the efficiency with which the compression is done. 
Furthermore, even though the performances no longer present a linear dependency with the number of chevrons, the best global results are, as in the previous case, given by the configuration with four chevrons.

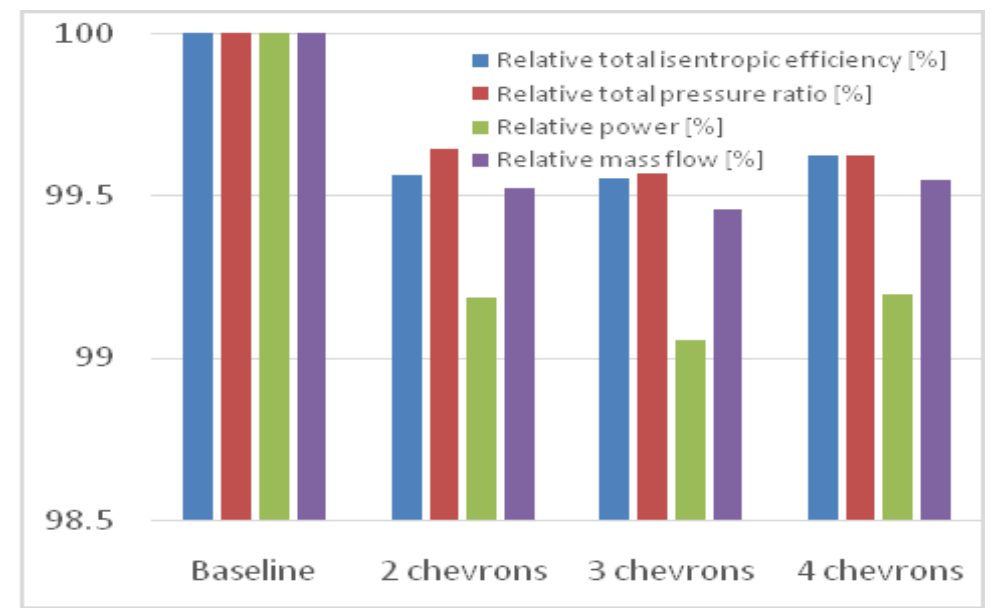

Fig. 6. Comparison between the functional parameters of the constant backpressure batch

In Fig. 7, the pressure distribution is presented, for the $20 \%, 50 \%$ and $80 \%$ span locations. For all chevron cases, the pressure loading increases slightly, particularly towards the leading edge on the pressure side. This trend is however compensated by the lower loading of the trailing edge side and the fact that overall, the suction side has a higher pressure (lower suction, per se).

Therefore, placing chevrons on the trailing edge shifts the pressure map to slightly lower pressure ratios. On the contrary, the efficiency map appears to be unaltered, as seen in the constant mass flow batch.

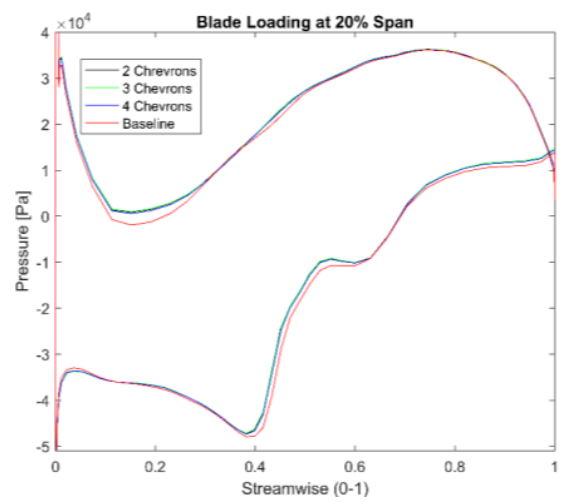

a) 


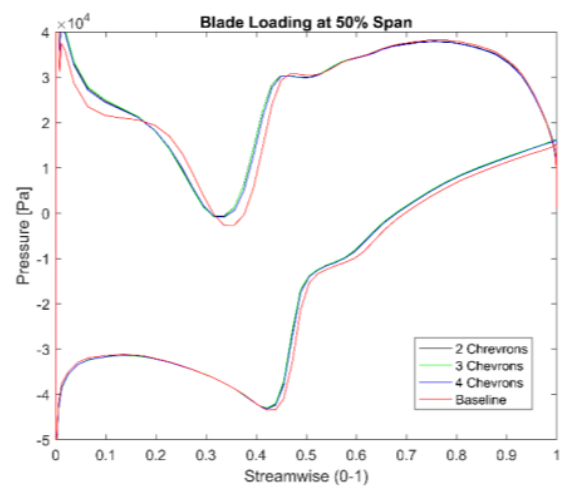

b)

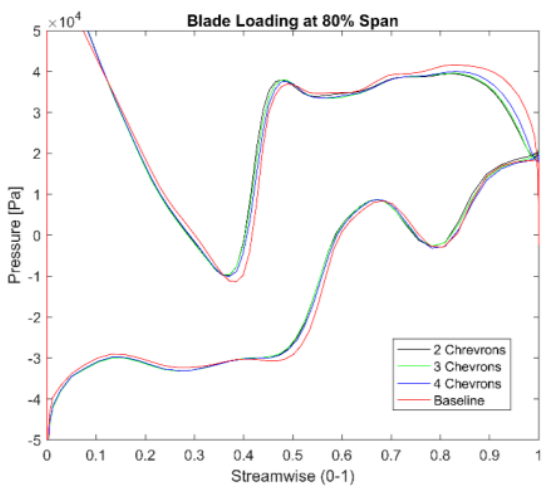

c)

Fig. 7. Blade loading across the span of the blade for the constant backpressure batch at:

a) $20 \%$ Span, b) $50 \%$ Span, c) $80 \%$ Span

\section{Conclusions}

The current paper explored the use of chevrons on the trailing edge of a highly loaded, high performance axial fan. NASA's Rotor 37 was selected and modified by cutting two, three and four chevrons into the trailing edge portion of the rotor near the tip of the blade. For simplicity, the rotor was considered to be shrouded in order to eliminate tip gap secondary flows that may interfere with the chevron induced secondary flow.

Two batches were considered, one with constant mass flow and one functional, with constant backpressure. To validate the computational methodology the results were compared with those given by NASA's technical report discussed in the introduction, with the data presented in Table 1. The second test case proved itself to be more accurate. 
The presence of chevrons influences loading across the entire blade span, even if the effect is more visible closer to the chevrons themselves.

On all cases pressure ratio dropped slightly, along with the power consumption. Counterintuitive, the efficiency was not altered in any of the cases, meaning that the small Reynolds number at which the chevrons function helps keep in check the pressure losses.

In the end, the use of chevrons appears to have a minimal impact on the functionality of the compressor, keeping the efficiency at a constant level while decreasing the pressure ratio by unloading the blades slightly. A more in-depth CFD study, covering the entire speedline would be a logical next step, along with the use of more sophisticated models such as DES or LES.

Also, in this paper the main emphasis is on the influence of chevron number and future work should concentrate on assessing the impact the geometrical characteristics of the chevrons have.

To conclude with, further work may also include a chevron-like trailing edge treatment such as the incorporation of serrated Gurney flaps which has been shown to increase pressure ratios of the rotor [16]. This would compensate the loading drop seen in the current paper. Also, chevron efficiency can be improved with unconventional devices such as sinusoidal chevrons [17] or rhino chevrons [18].

\section{References}

1. Biollo, R. and E. Benini. State-of-Art of Transonic Axial Compressors. Advances in Gas Trubine Technology, Italy, 2011. DOI: 10.5772/25257.

2. Dixon, S. L. Fluid mechanics, thermodynamics of turbomachinery. The University of Michigan, Pergamon Press, 1978, 263 p.

3. Samad, A., K. Y. Kim, T. Goel, R. T. Haftka, and W. Shyy. Multiple Surrogate Modeling for Axial Compressor Blade Shape Optimization. American Institute of Aeronautics and Astronautics: Jorunal of Propulsion and Power, 2008, 24, 2, 302-10. DOI: 10.2514/1.28999.

4. Reid, L. and R. D. Moore. Design and Overall Performance of Four Highly-Loaded, High-Speed Inlet Stages for an Advanced, High-Pressure-Ratio Core Compressor. Technical Report, NASA-TP-1337, 1978.

5. Reid, L. and R. D. Moore. Experimental Study of Low Aspect Ratio Compressor Blading. Technical Report, NASA-TM-79280, 1979.

6. Boretti, A. Experimental and Computational Analysis of a Transonic Compressor Rotor. Proc. of the $17^{\text {th }}$ Australasian Fluid Mechanics Conference, 2010, Auckland, New Zealand.

7. Chen, N., H. Zhang, Y. Xu, and W. Huang. Blade Parametrization and Aerodynamic Design Optimization for a 3D Transonic Compressor Rotor. Jorunal of Thermal Science, 2007, 16, 2, 105-14. 
8. Ahn, C. S. and K. Y. Kim. Aerodynamic Design Optimization of an Axial Flow Compressor Rotor. Proc. of ASME Turbo Expo 2002: Power for Land, Sea, and Air, 2002, 813-19. DOI:10.1115/GT2002-30445.

9. Dost, M., R. Jensen, and D. Meer. Fighting Noise in Gas Turbines. Noise reduction techniques in gas turbines. Fact sheet, 2016, AviationsFacts.eu.

10. Gliebe, P. R. Serrated Fan Blade. General Electric Company, 2004. United States Patent, US 6, 733, 240 B2.

11. Oro, J. M. F., A. Meana-Fernández, M. G. Vega, B. Pereiras and J. G. Pérez. LESbased simulation of the time-resolved flow for rotor-stator interactions in axial fan stages. International Journal of Numerical Mehtods for Heat \& Fluid Flow, 2019, 29, 2, 657-81.

12. Florean, F. G., A. C. Petcu, I. Porumbel and G. Dediu. PIV measurements in Low Noise Optimized Air Jet Pump Demonstrators. International Journal of Energy, 2016, 10, 33-43.

13. Gherman, B. G., I. Mălăel, M. Mihăescu and I. Porumbel. Jet Pump Optimization through Reynolds Averaged Navier-Stokes Simulation Analysis. AIAA $22^{\text {nd }}$ Computational Fluid Dynamics Conference, Dallas, TX, USA, 2015.

14. Jaron, R., A. Moreau, S. Guérin and R. Schnell. Optimization of Trailing-Edge Serrations to Reduce Open-Rotor Tonal Interaction Noise. Journal of Fluids Engineering, 2018, 140, 2. DOI:10.1115/1.4037981.

15. Juknevicius, A. and T. P. Chong. On the leading edge noise and aerodynamics of thin aerofoil subjected to the straight and curved serrations. Journal of Sound and Vibration, 2018, 425, 324-43. DOI:10.1016/j.jsv.2018.02.038.

16. Mălăel, I., H. Dumitrescu and A. Dumitrache. Methods for Improve the Performance of the Turbomachines Using the Flow Control. Proc. of the $9^{\text {th }}$ International Conference of Numerical Analysis and Applied Mathematics, 2011, 1389, 1. DOI:10.1063/1.3637913.

17. Chernem, F. J., J. E. Hammerberg, M. J. Andrews, V. Karkhanis and P. Ramaprabhu. On shock driven jetting of liquid from non-sinusoidal surfaces into a vacuum. Journal of Applied Physics, 2015, 118, 18. DOI:10.1063/ 1.4934645.

18. Drăgan, V. Contributions regarding the development of a highly aerodynamically efficient chevron for use in turbofan noise mitigation - the rhino chevron. Proc. of Caius Iacob 2011 International Conference, Bucharest, 2011. 